\title{
Massive extragalactic eclipsing binaries
}

\author{
Alceste Z. Bonanos \\ Carnegie Institution of Washington, 5241 Broad Branch Road, Washington, DC 20015, USA \\ email: bonanos@dtm.ciw.edu
}

Keywords. stars: binaries: eclipsing, stars: Wolf-Rayet, stars: individual (WR 20a)

Masses, radii and luminosities of distant stars can only be measured accurately in eclipsing binaries. The most massive eclipsing binary currently known is WR 20a, which consists of two $\sim 80 \mathrm{M}_{\odot}$ stars in a $3.7 \mathrm{~d}$ orbit. Analogs of WR 20a are bound to exist both in massive stellar clusters in our Galaxy and in nearby galaxies. The nearest ones are located in the clusters near the Galactic Center: the Center, Arches, and Quintuplet clusters. The severe amount of reddening in the galactic disk makes the study of galactic clusters challenging. However, with current 8-m class telescopes, the study of massive stars in nearby galaxies is also feasible. The nearest Local Group galaxies (LMC, SMC, M 31, M 33) provide the perfect laboratory for studying massive stars and determining their properties as a function of metallicity. Such studies will constrain models, confirm the dependence of evolution on metallicity and help understand the rate and nature of supernovae and gamma-ray bursts.

Finding massive binaries requires a variability survey from which the brightest and thus most massive eclipsing binaries are selected for spectroscopic follow-up. Given both light and radial velocity curves, one can determine the size of the orbit, the component radii and masses. Furthermore, by fitting synthetic spectra to the observed ones, one can infer the effective temperature, therefore the luminosity, and additionally solve for the distance. Several surveys have and are continuing to produce candidate massive binaries in nearby galaxies: the All Sky Automated Survey (Pojmanski 2002), the DIRECT Project surveying M31 and M33 for detached eclipsing binaries (see Bonanos et al. 2003, and references therein), the Araucaria project surveying nearby galaxies (e.g. NGC 6822 and NGC 300, Pietrzynski et al. 2004; Gieren et al. 2004) and the microlensing surveys towards the Magellanic Clouds and M31 (e.g. MACHO, OGLE, MEGA, POINT-AGAPE).

In this paper, I present preliminary results of a project to follow up the brightest eclipsing binaries found by OGLE in the LMC and SMC. Spectra were obtained over 13 nights using the Echelle spectrograph on the $2.5 \mathrm{~m}$ DuPont telescope and 2 nights on MIKE on the Clay Telescope at Las Campanas Observatory (LCO), Chile. Each binary was visited 4-10 times, depending on the number of nights during the observations that it was not in eclipse. Cross correlation with a template spectrum of a galactic early type star, excluding the blended Balmer lines was used to derive preliminary radial velocities for one of the LMC systems. The semi-amplitudes yielded minimum masses of $\sim 40 \mathrm{M}_{\odot}$ for the primary and $\sim 20 \mathrm{M}_{\odot}$ for the secondary, thus confirming that the brightest eclipsing binaries in the LMC do contain very massive stars. Future analysis with 2D cross correlation (Zucker et al. 1994) and spectral disentangling will provide more accurate velocities. This survey will yield accurate to $\sim 5 \%$ fundamental parameters for $\sim 20$ massive stars of low metallicity. 